\title{
Novel design of a fractional wavelet and its application to image denoising
}

\author{
Lanani Abderrahim ${ }^{1}$, Meghriche Salama ${ }^{2}$, Djouambi Abdelbaki $^{3}$ \\ ${ }^{1}$ Department of Electronics, University of Batna 2, Mostefa Ben Boulaid, Batna, Algeria \\ ${ }^{2}$ Department of Pharmaceutical Engineering, University of Constantine 3, Salah Boubnider, Constantine, Algeria \\ ${ }^{3}$ Department of Electrical Engineering, Larbi Ben M’hidi University, Oum El Bouaghi, Algeria
}

\begin{tabular}{l}
\hline Article Info \\
\hline Article history: \\
Received Feb 23, 2019 \\
Revised Jun 2, 2019 \\
Accepted Sep 26, 2019 \\
\hline
\end{tabular}

Keywords:

Fractionaldelay

Fractionalfilters

Image denoising

The Haar wavelet

\begin{abstract}
This paper proposes a new wavelet family based on fractional calculus. The Haar wavelet is extended to fractional order by a generalization of the associated conventional low-pass filter using the fractional delay operator $Z^{-D}$. The high-pass fractional filter is then designed by a simple modulation of the low-pass filter. In contrast, the scaling and wavelet functions are constructed using the cascade Daubechies algorithm. The regularity and orthogonality of the obtained wavelet basis are ensured by a good choice of the fractional filter coefficients. An application example is presented to illustrate the effectiveness of the proposed method. Thanks to the flexibility of the fractional filters, the proposed approach provides better performance in term of image denoising.
\end{abstract}

This is an open access article under the $\underline{C C B Y-S A}$ license.

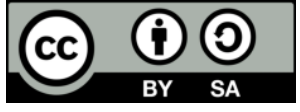

\section{Corresponding Author:}

Lanani Abderrahim,

Department of Electronics,

University of Batna 2, Mostefa Ben Boulaid, Batna, Algeria .

Email: lanani_arahim@yahoo.fr

\section{INTRODUCTION}

The main purpose of signal processing is to describe signals related to the real world for the purpose of processing, identifying, compressing, understanding or transmitting. In this context, wavelets have always played a very important role, and we no longer count the applications that use them. It is a tool allows analyzing and locating the discontinuities of a signal along one or two dimensions and at different scales. This feature is used to solve many problems, such as image compression and restoration [1].

Since their introduction, the wavelets have been very rapidly developed and have attracted the attention of many researchers. Solid mathematical foundations (basis) were quickly put into place for the notions of an orthogonal basis [2], multiresolution analysis [3-5] and wavelets with compact support [6], giving birth to several real, complex and fractional wavelet families [1]. However, identification of an adequate analyzing wavelet remains an important problem. No single wavelet has been adapted to all cases. For some applications, it is possible that no suitable wavelet exists among the known wavelets. In such cases, it is necessary to try to build a new wavelet adapted to the specific problem to be addressed.

Thus, many studies have appeared whose purpose is to build wavelets which have more elegant mathematical properties and are more efficient. Among the wavelets which are currently of considerable interest those based on fractional calculus. The main advantage of having a fractional order is flexibility, which allows adjustments in transform parameters such as regularity and localization of the base functions [5]. These advantages can be exploited to improve several approaches and ameliorate problems such as denoising by thresholding wavelet coefficients [1]. The idea of fractional wavelets was first proposed 
by Mendlovic [7] in the context of optical signal processing, where they were defined as a cascade of the classical wavelet transform and the fractional Fourier transform (FRFT) [8]. Shortly thereafter, Huang and Suter [9] proposed a fractional version of the wave packet transform (FRWPT), based on combining FRFT and the wave packet transform (WPT). Due to a lack of physical interpretation and the complexity of the calculation, the FRWPT unfortunately did not receive much attention. However, several years later, the idea was extended in the context of spline wavelets to non-integer degrees [10]. Quincunx wavelets have also been generalized to non-integer orders with a construction based on fractional Quincunx filters, which are generated through the diamond McClellan transform [11]. Several fractional wavelet models have recently been proposed and developed, with a wide range of applications [12-16].

The aim of the present paper is to introduce the new fractional wavelet (NFRW), which we consider a generalization of the Haar wavelet [1]. However, this idea can also be extended to genera lize other wavelet families. The construction generally begins with the choice of a low-pass digital filter with the orthogonality property checked in Fourier or direct space; then it is generalized through the fractional delay (FD) $Z^{-D}$ [17] while ensuring correct properties of orthogonality, compact support and regularity. The high-pass filter can then be built from the low-pass filter, and the associated scaling function and wavelet can be deduced. To illustrate the potential of the proposed wavelet, we present a denoising application on images. The rest of this paper is organized as follows. Section 2 briefly defines the discrete wavelet transform (DWT) and introduces the relation between multiresolution analysis and filter banks. Section 3 proposes the NFRW. Section 4 examines and simulates an experimental application of the NFRW. Finally, section 5 presents concluding remarks.

\section{DISCRETE WAVELET TRANSFORM AND FILTER BANKS}

The DWT is a multiresolution/multifrequency representation. This tool separates the data, functions or operators in frequency components according to a resolution adapted to the scale. The multiresolution analysis developed by Mallat [5] connects the DWT and filter banks in an elegant way, with the DWT considered as a process of decomposing the signal into approximations and details. The original signal $\tilde{x}[\mathrm{n}]$ passes through two complementary low-pass and high-pass filters $\tilde{h}[\mathrm{n}]$ and $\tilde{g}[\mathrm{n}]$, followed by a factor 2 subsampling, and emerges as two signals: the approximation signal $\mathbf{A}$ and the detail signal $\mathbf{D}$, respectively [5]. Figure 1 illustrates this principle. The reconstruction is performed by low-pass and high-pass synthesis filters $\bar{h}[\mathrm{n}]$ and $\bar{g}[\mathrm{n}]$, preceded by a factor 2 upsampling, as illustrated in Figure 2 .

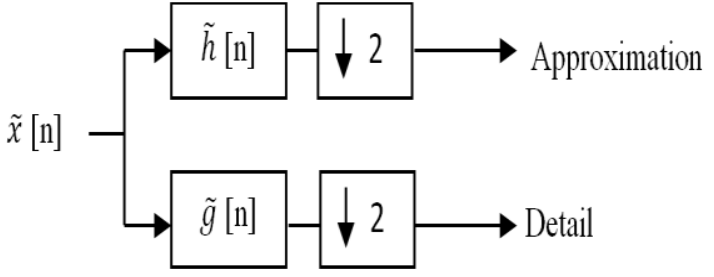

Figure 1. One-dimensional wa velet decomposition

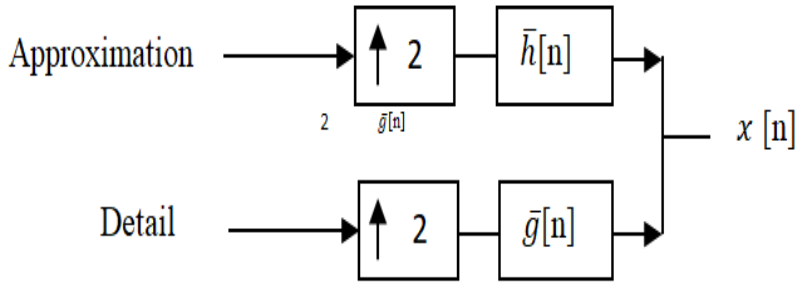

Figure 2. One-dimensional wa velet reconstruction

The filter bank ensures a perfect reconstruction if and only if [18]

$$
\left\{\begin{array}{c}
\tilde{H}(\omega+\pi) \bar{H}(w)+\widetilde{G}(\omega+\pi) \bar{G}(\omega)=0, \\
\widetilde{H}(\omega) \bar{H}(\omega)+\widetilde{G}(\omega) \bar{G}(\omega)=2,
\end{array}\right.
$$

Where $\widetilde{H}, \widetilde{G}, \bar{H}$ and $\bar{G}$ are, respectively, the transfer functions of the low-pass and high-pass analysis and the synthesis filters. In the case of an orthogonal basis, the high-pass filter is a modulated version of the low-pass filter; however, the analysis and synthesis filters are identical up to a centralsymmetry. 
Moreover, the orthogonal wavelet transform is generally characterized by a scaling function $\boldsymbol{\varphi}$ and wavelet $\boldsymbol{\psi}$ (for more details see [19]), where the scaling function can be expressed in terms of the low-pass filter by the equation

$$
\varphi(x)=\sum_{n} \tilde{h}[n] \sqrt{2} \varphi(2 x-n) .
$$

In contrast, the wavelet function decomposes at the lower scale based on the scaling functions and can be expressed in terms of the high-pass filter by the equation

$$
\psi(x)=\sum_{n} \tilde{g}[n] \sqrt{2} \varphi(2 x-n) .
$$

\section{PROPOSED DISCRETE FRACTIONAL WAVELET STRUCTURE}

From a structural point of view, the DWT is identical to an iterated filter bank, which gives it a multiresolution character, so we can use a fractional filter to implement a fractional discrete wavelet as shown in Figure 3; indeed, based on fractional operators, we can build fractional filters which lead to wavelets with interesting properties (precision, flexibility, regularity, etc.).

In this paper, we have chosen the Haar filter as the starting point for our construction; this is an orthogonal FIR filter which has a linear phase, one vanishing moment and a finite support. It is generalized using a fractional delay (FD) $Z^{-D}$, which is simulated by a filter which we call the "fractional delay filter" (FDF). We can then build the high-pass fractional filter from the low-pass fractional filter and deduce the associated scaling function and wavelet by the cascade algorithm [6]. A perfect reconstruction is ensured by fractionalsynthesis filters, built in accordance with the conditions of (1).

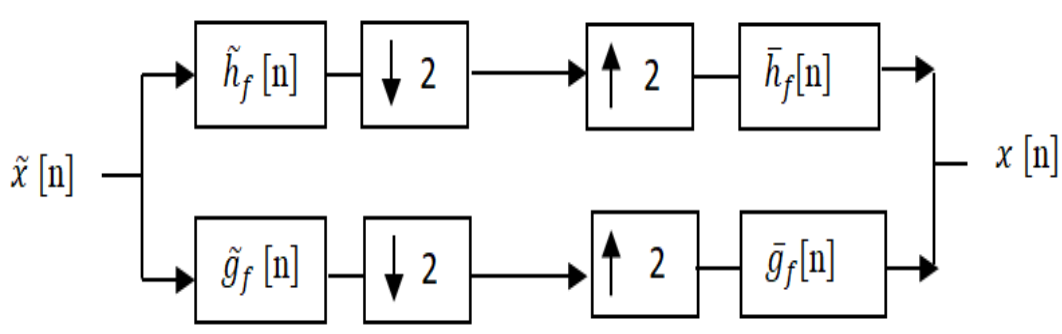

Figure 3. One-dimensional fractional wa velet decomposition and reconstruction

\subsection{Fractional filter design}

The transfer function of the low-pass filter associated with the Haar analysis is described by the equation [20]

$$
\widetilde{H}(z)=\frac{\left(1+z^{-1}\right)}{\sqrt{2}}
$$

We propose a generalization of this function to a fractional order by exploiting the fractional operator $Z^{-D}$ with $D$ considered to be a real number:

$$
\widetilde{H_{f}}(z)=\frac{\left(1+z^{-D}\right)}{\sqrt{2}}
$$

This function can be written in a more general way as follows:

$$
\widetilde{H_{f}}(z)=A+B \cdot Z^{-D}
$$

where $D \in \mathbb{R}$ is the filter order, and $A \in \mathbb{R}$ and $B \in \mathbb{R}$ are the coefficients. The orthogonality and regularity of the scaling function and wavelet are ensured by a good choice of the coefficients $A$ and $B$. In the classical case, $D=1, A=1 / \sqrt{2}$ and $B=1 / \sqrt{2}$. 
In contrast, it should be noted that the fractional order digital delay $Z^{-D}$ is represented by an irrational function in which the impulse response is infinitely long. This is similar to a non-causal filter for any finite shift in time, which makes its implementation an unrealizable task [17] and implies a very limited implementation for the transfer function of the fractional filter $\widetilde{H}_{f}$. Therefore, it is necessary to use approximation techniques to obtain a realizable implementation of the FD and, obviously, a rational representation for the transfer function of the fractional filter $\widetilde{H}_{f}$. The majority of the design techniques which have been proposed in the literature to approximate the FD focus exclusively on finite impulse response (FIR) and infinite impulse response filters (IIR) [17]. In this paper, we focus on the FIR filter due to its easy implementation and good approximation.

\subsubsection{Fractional delay approximation using FIR filters}

The transferfunction of the FIR filter approximating the FD $Z^{-D}$ is given by [17]:

$$
H(Z)=\sum_{n=0}^{N} h[n] Z^{-n},
$$

where $N$ is the order of the filter and $h[\mathrm{n}], n=0,1, \ldots N$, are the real coefficients which form the impulse response of the FIR filter.

Several techniques have been developed to identify the coefficients $h[\mathrm{n}]$, such as windowing methods, maximally flat FIR approximation (Lagrange interpolation), FIR filter design with a smooth transition band function, the minimax or Chebyshev method, and the stochastic method. More details a bout these can be found in [17]. In the present paper, we choose the method based on Lagrange interpolation, due to the ease of calculating filter coefficients from a closed form equation and also because the filter obtained by this method has a completely flat magnitude frequency response at low frequencies. Indeed, the Lagrange interpolation is one of the simplest and most efficient methods for designing an FIR filter approximating the FD $Z^{-D}$. The FDF coefficients are given by [17]:

$$
h[n]=\prod_{\substack{k=0 \\ k \neq n}}^{N} \frac{D-k}{n-k} \quad \text { for } n=0,1,2, \ldots N
$$

where $N$ is the order of the filter and $D$ is the desired delay,

$$
\frac{N-1}{2} \leq D \leq \frac{N+1}{2} \quad \text { for odd } N
$$

such that

$$
\frac{N}{2}-1 \leq D \leq \frac{N}{2}+1 \quad \text { for even } N
$$

Using (7), we draw up Table 1, which groups the coefficients for Lagrange FD filters of order $N=1,2$ and 3 .

Table 1. The coefficients of the Lagrange fractionaldelay filters of order $N=1,2$ and 3

\begin{tabular}{ccccc}
\hline & $h[0]$ & $h[1]$ & $h[2]$ & $h[3]$ \\
\hline$N=1$ & $1-D$ & $D$ & & \\
$N=2$ & $(D-1)(D-2) / 2$ & $-D(D-2)$ & $D(D-1) / 2$ & \\
$N=3$ & $-(D-1)(D-2)(D-3) / 6$ & $D(D-2)(D-3) / 2$ & $-D(D-1)(D-3) / 2$ & $D(D-1)(D-2) / 6$ \\
\hline
\end{tabular}

\subsubsection{Fractional filter bank approximation}

Following the approximation of the FD $Z^{-D}$ by an FIR filter, the transfer function of the fractional filter $\widetilde{H_{f}}(6)$ is represented by the following function:

$$
\widetilde{H_{f}}(z) \approx A+B \cdot\left(\sum_{n=0}^{N} h[n] Z^{-n}\right) .
$$

Where $h(n)$ and $N$ are respectively the coefficients and the order of the FIR filter approximating the FD $Z^{-D}$, and $A$ and $B$ are the coefficients of the fractional filter which constitutes the wavelet. In order to ensure the orthogonality and regularity of the scaling function and the wavelet, the coefficients $A$ and $B$ are chosen according to the following conditions [2-3], [21]: 


$$
\begin{aligned}
& \forall \omega \in \mathbb{R},\left|\widetilde{H_{f}}(\omega)\right|^{2}+\left|\widetilde{H_{f}}(\omega+\pi)\right|^{2}=2, \\
& \widetilde{H_{f}}(0)=\sqrt{2}, \\
& \left|\widetilde{H_{f}}(\pi)\right|=0 .
\end{aligned}
$$

Condition (11) is a constraint corresponding to the orthonormality of the scaling function, and it also means that the filter $\widetilde{H_{f}}$ is a conjugate mirror filter [22]. Condition (12) is a simple normalization, and condition (13) ensures the regularity of the scaling functions and wavelets. There exist only a few solutions for identifying $A$ and $B$ while respecting these three conditions. In this paper, we calculate $A$ and $B$ while ensuring that conditions (12) and (13) hold; however, condition (11) is provided only approximately, i.e.:

$$
\begin{aligned}
& \left|\widetilde{H_{f}}(\omega)\right|^{2}+\left|\widetilde{H_{f}}(\omega+\pi)\right|^{2} \approx 2, \\
& \left|\widetilde{H_{f}}(\omega)\right|^{2}+\left|\widetilde{H_{f}}(\omega+\pi)\right|^{2}-2=\varepsilon .
\end{aligned}
$$

The coefficients $A$ and $B$ of the fractionalfilter $\widetilde{H_{f}}$ are given in Table 2 for $N=1,2$ and 3 .

The error $\varepsilon$ is very small and does not have dramatic effects on our construction, while the fractional filter $\widetilde{H_{f}}$ generates regular orthogonal bases for $0.5<D<1.7$, which implies a limitation of the FD order to the interval $[0.5,1.7]$. Beyond this interval, the accuracy of the calculation can cause problems, and the orthogonality of the obtained bases is lost.

Table 2. Fractionalfilter coefficients for different values of $N$

\begin{tabular}{ccc}
\hline & $\mathrm{A}$ & $\mathrm{B}$ \\
\hline \multirow{2}{*}{$N=\mathbf{1}$} & $\sqrt{2}-\frac{\sqrt{2}}{1-(h[0]-h[1])}$ & $\frac{\sqrt{2}}{1-(h[0]-h[1])}$ \\
$N=\mathbf{2}$ & $\sqrt{2}-\frac{\sqrt{2}}{1-(h[0]-h[1]+h[2])}$ & $\frac{\sqrt{2}}{1-(h[0]-h[1]+h[2])}$ \\
$N=3$ & $\sqrt{2}-\frac{\sqrt{2}}{1-(h[0]-h[1]+h[2]-h[3])}$ & $\frac{1-(h[0]-h[1]+h[2]-h[3])}{1-h}$ \\
\hline
\end{tabular}

According to the above table, the fractional filter $\widetilde{H_{f}}$ is represented by a finite number of non-zero coefficients, i.e. it is a filter with compact support, where the number of coefficients is directly related to the FD filter order (length of filter $\widetilde{H_{f}}=\mathrm{FD}$ filter order +1 ). The regularity of the scaling function and the wavelet are ensured as long as the zero of $\widetilde{H_{f}}(\omega)$ at $\omega=\pi$ has an order of at least 1 [21].

On the other hand, as indicated above (in section 2), the high-pass fractional filter is a modulated version of the low-pass filter, where the transfer function is defined by the property of quadrature mirror filters [23]; its coefficient is given by the following expression:

$$
\widetilde{g_{f}}[n]=(-1)^{N-n} \widetilde{h_{f}}[N-n]
$$

However, the synthesis filters ( $H$ and $G$ ) are constructed according to (1), and their coefficients are defined as follows:

$$
\begin{aligned}
& \bar{h}_{f}[n]=\widetilde{h_{f}}[N-n], \\
& \bar{g}_{f}[n]=\widetilde{g_{f}}[N-n] .
\end{aligned}
$$

The frequency responses of the analysis and synthesis filters $(\widetilde{H}, \widetilde{G}, \bar{H}$ and $\bar{G})$ are adjusted by the variation of the parameter $D$, as shown in Figure 4. It can be seen that the generalization of these filters through the fractional operator $Z^{D}$ leads to more flexible filters with better precision, allowing a continuous adjustment of the key parameters of the filters. 


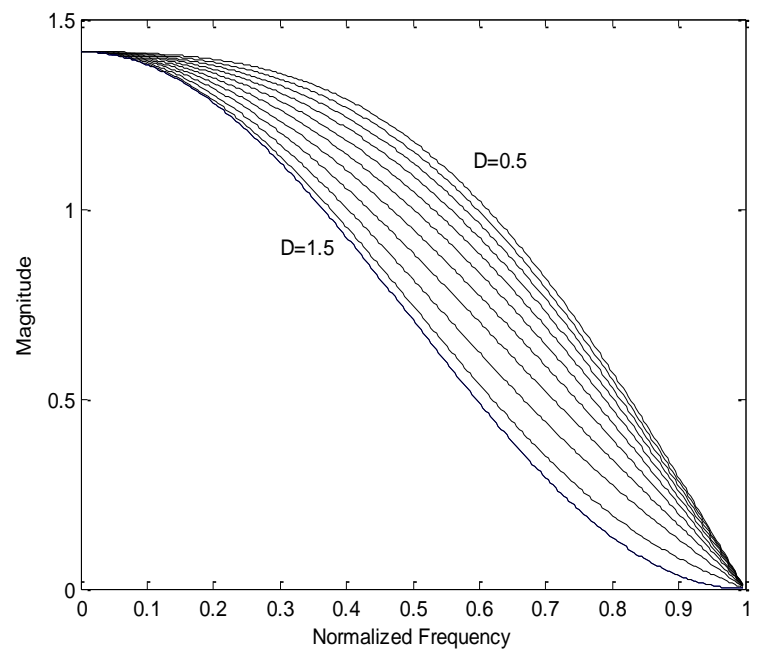

(a)

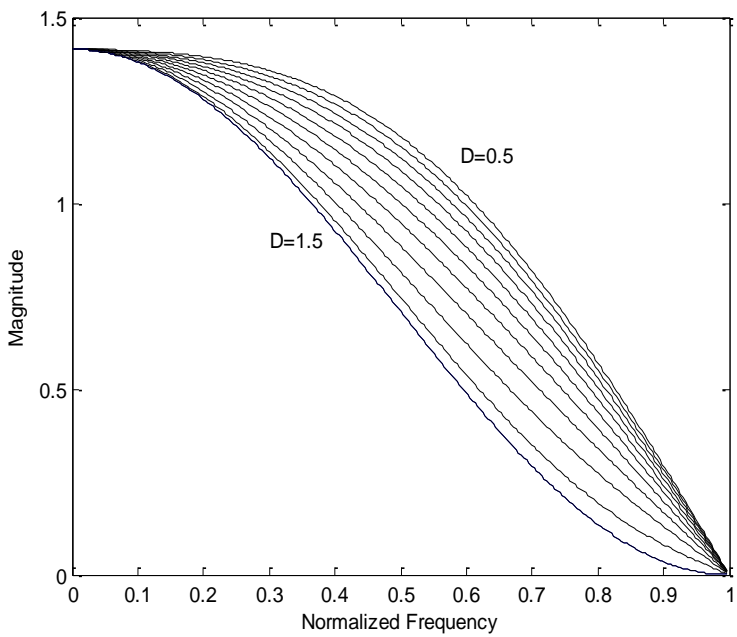

(c)

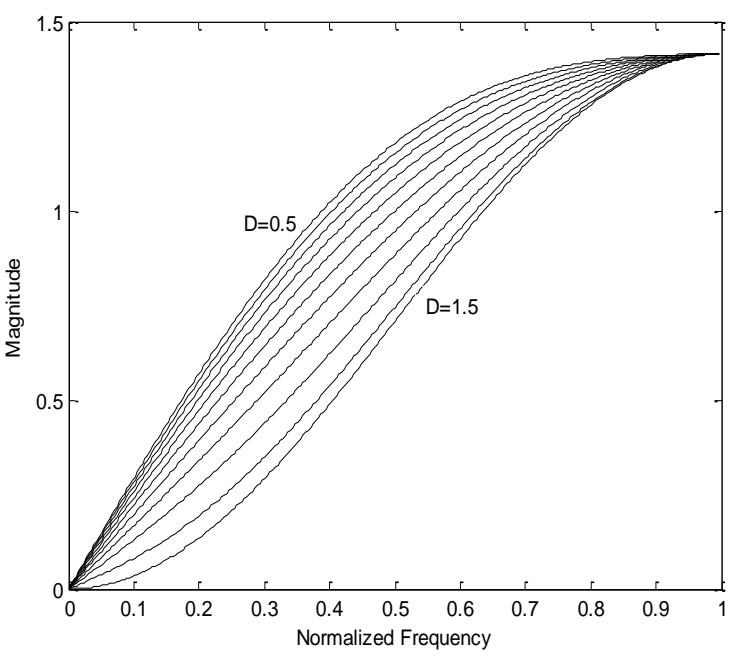

(b)

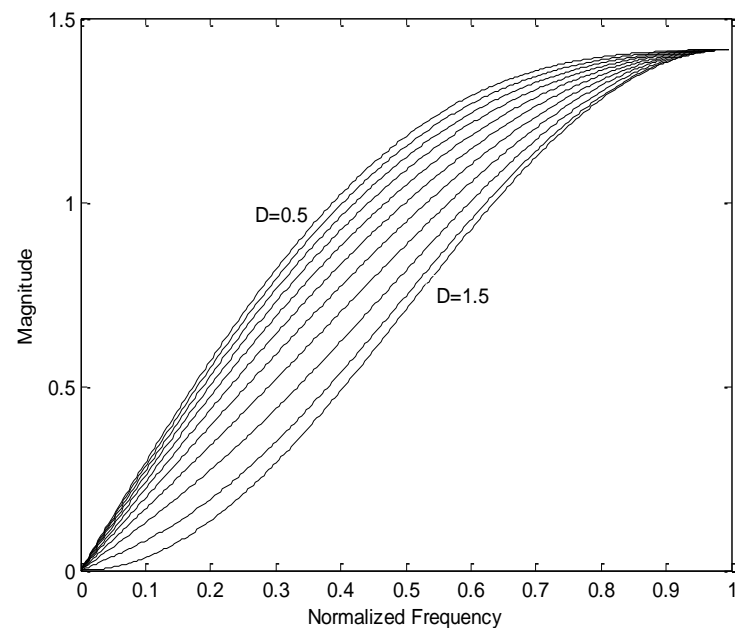

(d)

Figure 4. Frequency responses of analysis and synthesis filters for $N=2$ and $D=0.5$ 1.5: (a) low-pass analysis filter, (b) high-pass analysis filter, (c) low-pass synthesis filter, (d) high-pass synthesis filter

\subsection{Fractional scaling function and fractional wavelet}

The construction of the scaling and wavelet functions is ensured by the cascade algorithm [6]. They are respectively represented in terms of the low-pass and high-pass filters $\tilde{h}_{f}$ and $\tilde{g}_{f}$ by the following expressions:

$$
\begin{aligned}
& \varphi_{f}(x)=\sum_{n} \tilde{h}_{f}[n] \sqrt{2} \varphi_{f}(2 x-n), \\
& \psi_{f}(x)=\sum_{n} \tilde{g_{f}}[n] \sqrt{2} \varphi_{f}(2 x-n) .
\end{aligned}
$$

Figure 5 presents the scaling and wavelet functions for different values of $D: 0.5,0.8,1,1.1,1.3$ and 1.5 . It can be seen that these functions gain in regularity with the increase of the parameter $D$, until the value $D=1.7$, where the orthogonality of the obtained base is completely lost. 

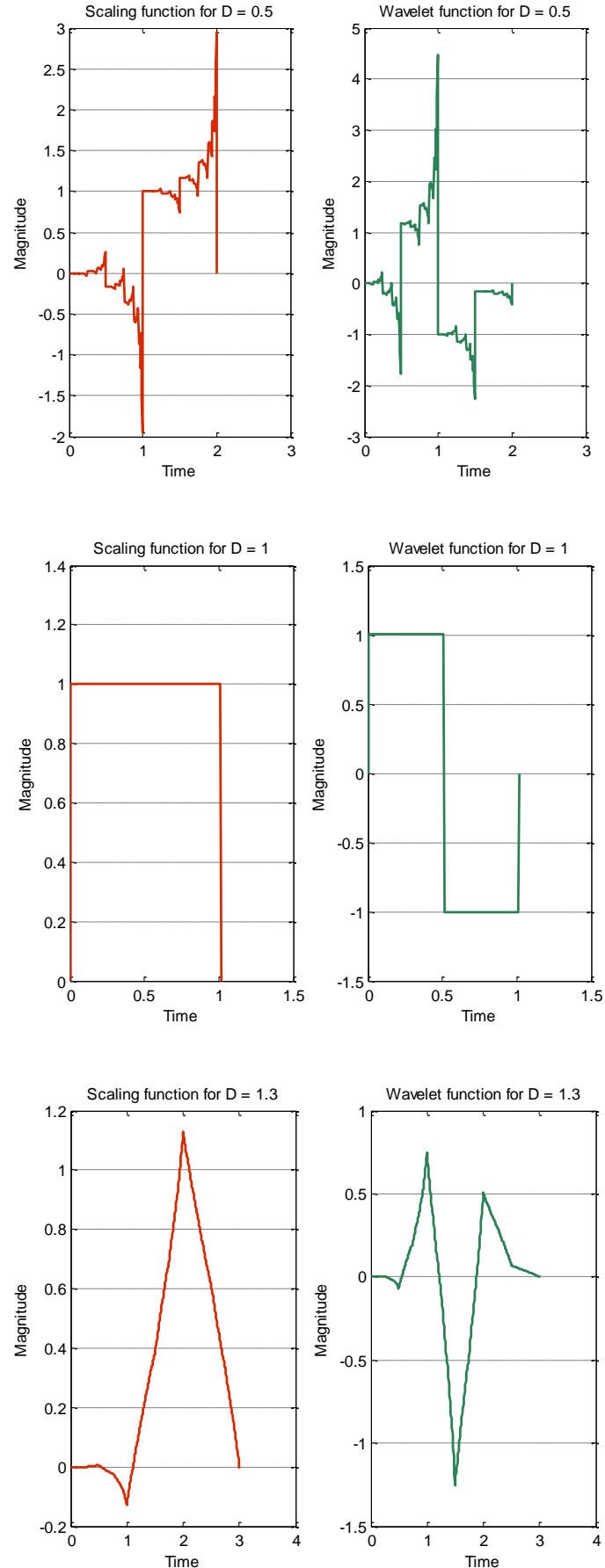
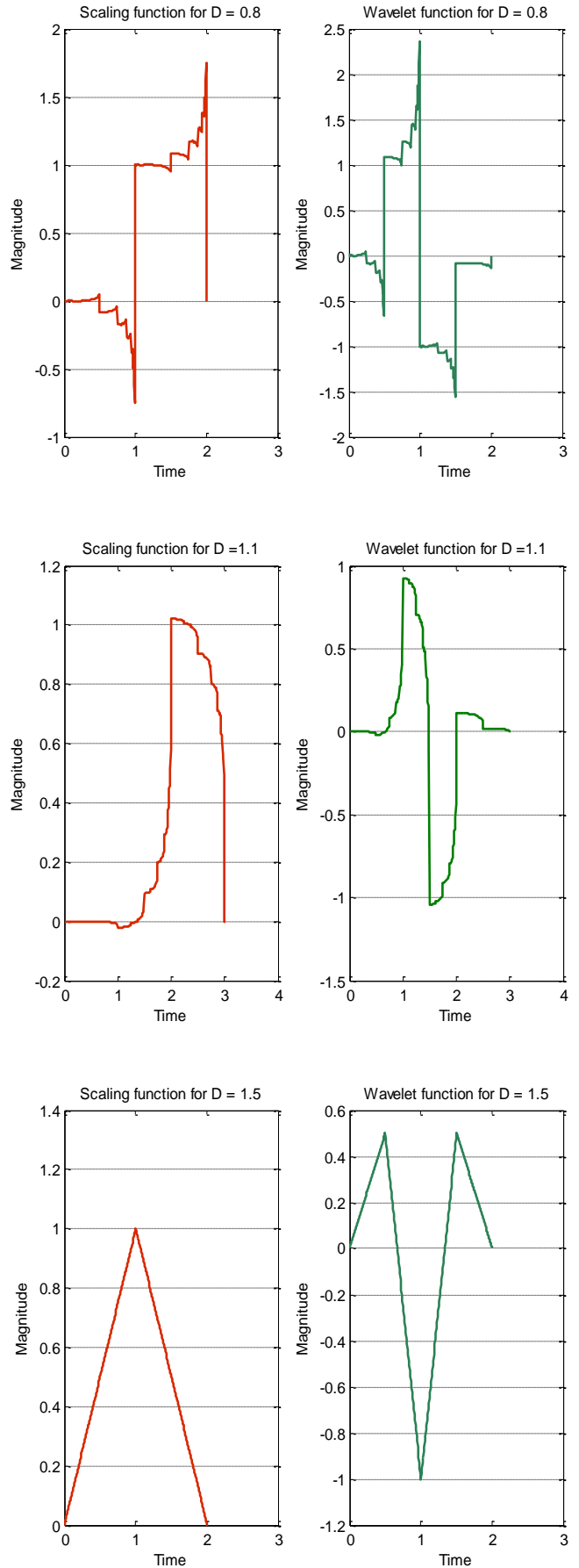

Figure 5. The fractional scaling and wavelet functions for different values of D

\section{APPLICATION AND RESULTS}

In this section, to illustrate the potential of the proposed wavelet, some results of using the proposed fractional wavelet in image denoising algorithm are presented. The denoising algorithms, which threshold wavelet coefficients, are based on an estimation of the noise variance. This estimation is obtained according to a statistical calculation performed on the wavelet coefficients at the first scale. The threshold computed from this estimation is then applied at each scale to denoise the image (for more details see [24]).

In the context of dyadic analysis, an adaptive threshold selection using Stein's unbiased risk estimate (SURE) principle and hard thresholding [25], the denoising algorithm is applied to a set of 8 -bit gray scale images: Lena, boat, cameraman and building as shown in Figure 6, corrupted by simulated additive Gaussia $n$ 
white noise with standard deviations of 10,20 and 30. The wavelet basis proposed in this paper (generalized Haar) is exploited for different values of $D$, where the ordinary case is $D=1$.
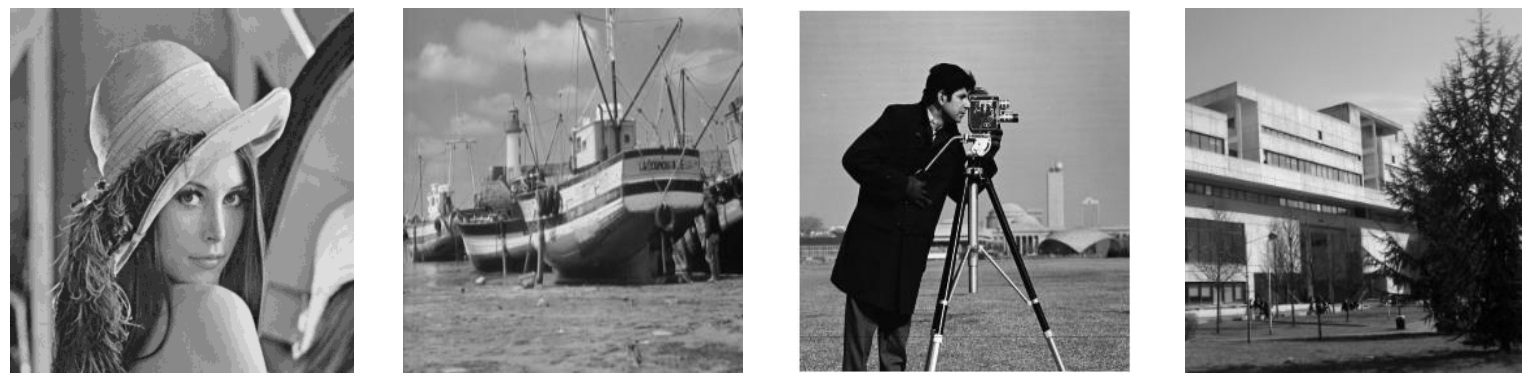

Figure 6. Input images (Lena, boat, ca mera man and building)

To evaluate and measure the quality of the restoration, the peak signal-to-noise ratio (PSNR) is computed (with MATLAB ${ }^{\circledR}$ coding). It is defined for a $B$-bit gray scale level by the equation

$$
P S N R=10 \log _{10}\left(\frac{\left(2^{B}-1\right)^{2}}{M S E}\right)
$$

where $M S E$ is the mean square error between the initial image (without noise) and the denoised image. The PSNR results after denoising are reported in Table 3, for different values of the parameter $D$. Figure 7 presents the PSNR variations of the Lena, building, cameraman and boat image denoising with three different noise levels using the proposed wavelet basis with different values of $D$.

Table 3. PSNR (dB) results of denoising images with Gaussian noise with standard deviations $\sigma=10,20$ and

\begin{tabular}{|c|c|c|c|c|c|c|c|c|c|c|c|c|}
\hline \multicolumn{13}{|c|}{ PSNR denoised image $(\mathrm{dB})$} \\
\hline $\begin{array}{l}\text { Input } \\
\text { image }\end{array}$ & $\begin{array}{c}\sigma / \text { PSNR } \\
\text { noised } \\
\text { image }\end{array}$ & $\mathrm{D}=0.5$ & $\mathrm{D}=0.6$ & $\mathrm{D}=0.7$ & $\mathrm{D}=0.8$ & $\mathrm{D}=0.9$ & $\mathrm{D}=1$ & $\mathrm{D}=1.1$ & $\mathrm{D}=1.2$ & $\mathrm{D}=1.3$ & $\mathrm{D}=1.4$ & $\begin{array}{l}D=1.5 \\
D=1.6\end{array}$ \\
\hline \multirow{3}{*}{$\begin{array}{l}\text { Lena } \\
512 \times 5 \\
12\end{array}$} & $\sigma=10 / 20.00$ & 21.89 & 22.16 & 22.86 & 23.44 & 24.15 & 24.95 & 26.23 & 27.36 & 27.81 & 28.13 & $\begin{array}{l}28.43 \\
27.65\end{array}$ \\
\hline & $\sigma=20 / 13.98$ & 16.58 & 17.00 & 17.60 & 18.25 & 18.93 & 18.93 & 21.10 & 22.29 & 22.88 & 23.43 & $\begin{array}{l}23.86 \\
22.89\end{array}$ \\
\hline & $\sigma=30 / 10.46$ & 13.21 & 13.74 & 14.45 & 15.22 & 15.98 & 15.98 & 17.77 & 19.00 & 19.51 & 20.18 & $\begin{array}{l}20.67 \\
19.56\end{array}$ \\
\hline \multirow{3}{*}{$\begin{array}{c}\text { Buildi } \\
\text { ng } \\
512 \times 5 \\
12\end{array}$} & $\sigma=10 / 20.00$ & 18.64 & 18.84 & 19.33 & 20.08 & 21.28 & 22.60 & 23.36 & 23.69 & 23.83 & 23.71 & $\begin{array}{l}23.81 \\
23.58\end{array}$ \\
\hline & $\sigma=20 / 13.98$ & 15.55 & 16.22 & 16.73 & 17.29 & 17.99 & 18.72 & 19.82 & 17.14 & 21.04 & 21.35 & $\begin{array}{l}21.59 \\
21.01\end{array}$ \\
\hline & $\sigma=30 / 10.46$ & 13.13 & 13.62 & 13.62 & 14.87 & 15.51 & 15.85 & 17.14 & 18.20 & 18.61 & 19.11 & $\begin{array}{l}19.43 \\
18.63\end{array}$ \\
\hline \multirow{3}{*}{$\begin{array}{c}\text { Came } \\
\text { raman } \\
256 \times 2 \\
56\end{array}$} & $\sigma=10 / 20.02$ & 18.29 & 18.81 & 19.65 & 20.20 & 20.92 & 23.18 & 24.15 & 24.59 & 24.61 & 24.36 & $\begin{array}{l}24.46 \\
21.30\end{array}$ \\
\hline & $\sigma=20 / 13.97$ & 15.81 & 16.28 & 16.91 & 17.40 & 18.07 & 18.07 & 20.12 & 21.05 & 21.32 & 21.66 & $\begin{array}{l}21.94 \\
18.74\end{array}$ \\
\hline & $\sigma=30 / 10.45$ & 13.46 & 13.54 & 14.11 & 14.89 & 15.52 & 15.52 & 17.28 & 18.44 & 18.71 & 19.31 & $\begin{array}{l}19.63 \\
18.74\end{array}$ \\
\hline \multirow{3}{*}{$\begin{array}{c}\text { Boat } \\
512 \times 5 \\
12\end{array}$} & $\sigma=10 / 20.00$ & 20.87 & 21.64 & 21.79 & 22.55 & 23.06 & 24.22 & 25.29 & 26.10 & 26.39 & 26.51 & $\begin{array}{l}26.71 \\
26.18\end{array}$ \\
\hline & $\sigma=20 / 13.98$ & 16.35 & 16.79 & 17.44 & 17.94 & 18.64 & 19.44 & 20.77 & 21.88 & 22.31 & 22.82 & $\begin{array}{l}23.18 \\
22.30\end{array}$ \\
\hline & $\sigma=30 / 10.46$ & 13.04 & 13.55 & 14.24 & 15.04 & 15.81 & 16.21 & 17.60 & 18.83 & 19.30 & 19.92 & $\begin{array}{l}20.36 \\
19.33 \\
\end{array}$ \\
\hline
\end{tabular}



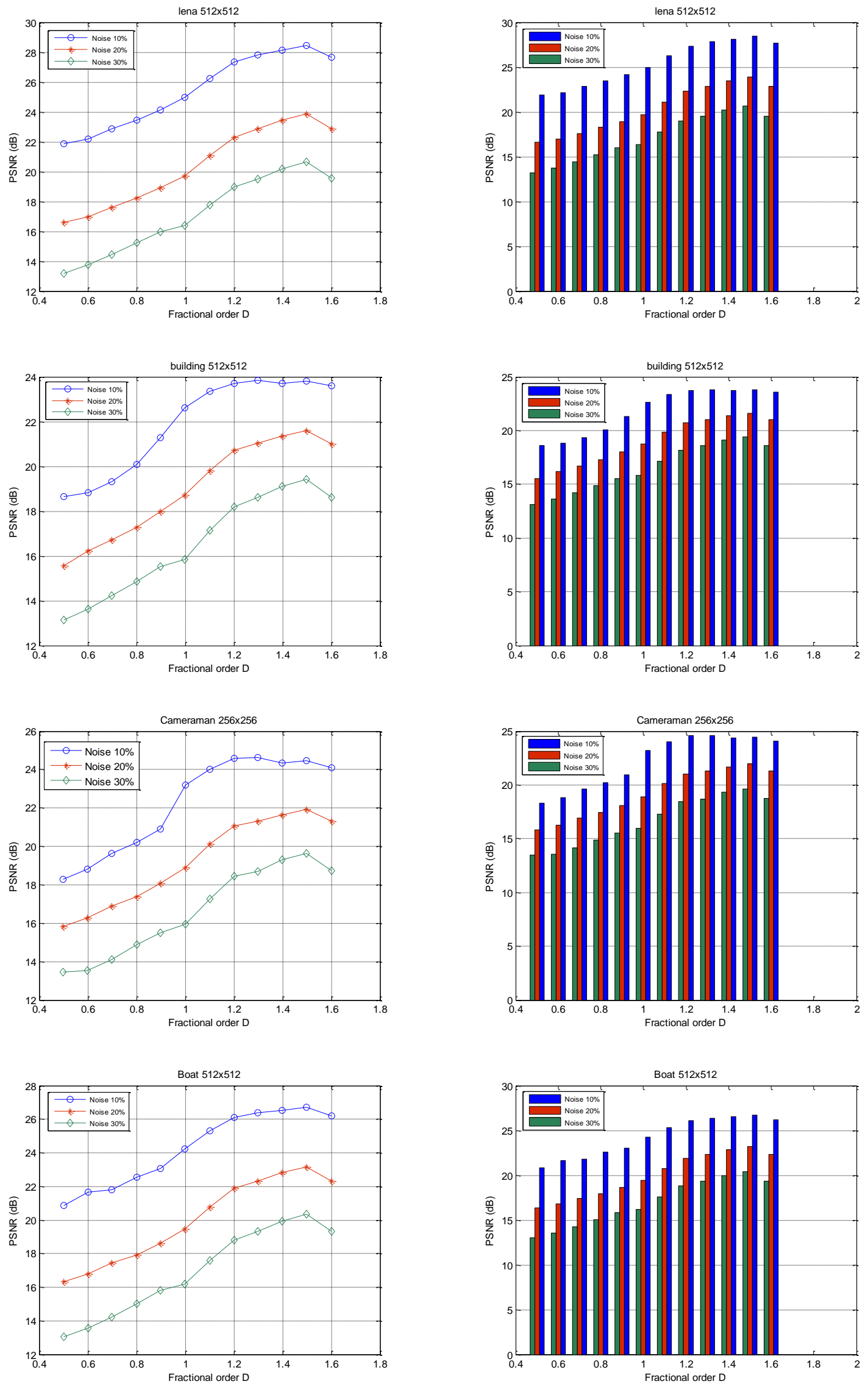

Figure 7. Presents the PSNR variations of the Lena, building, cameraman and boat image denoising 
As can be seen, the best value of the PSNR for all images with different noise densities corresponds to a fractionalvalue of the parameter $\mathrm{D}$, which indicates that the fractional analysis leads to an improvemen $\mathrm{t}$ in the denoising result due to the flexibility of the fractional filters which constitute the proposed wavelet basis. Moreover, it can be seen in the denoising results for the images Lena, boat, cameraman and building as shown in Figure 8 that the use of fractional calculation through the FD $z^{-D}$ offers considerable performance improvement in terms of visual quality, especially because the noise is eliminated with significant preservation of edge sharpness.

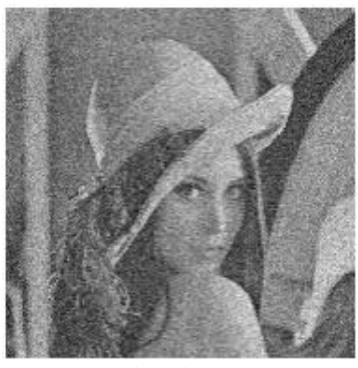

Noisy image

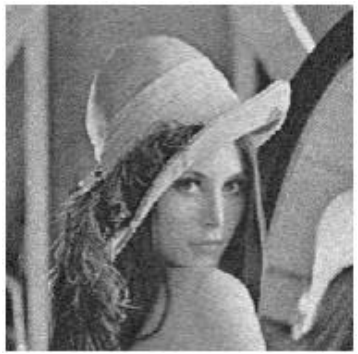

$\mathrm{D}=1.1$

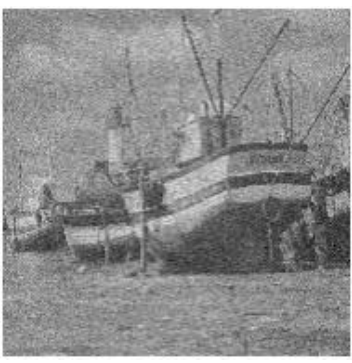

Noisy image

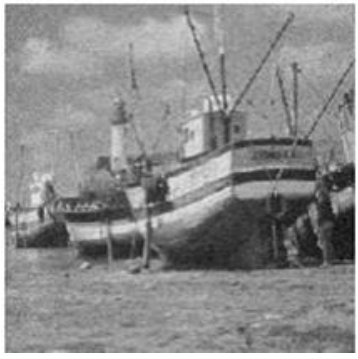

$\mathrm{D}=1.1$

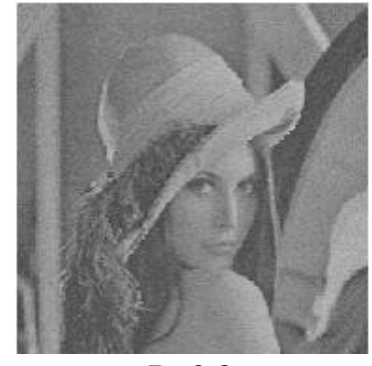

$\mathrm{D}=0.8$

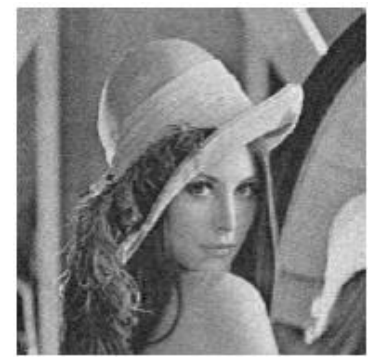

$\mathrm{D}=1.3$

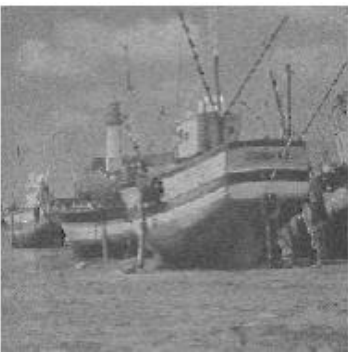

$\mathrm{D}=0.8$

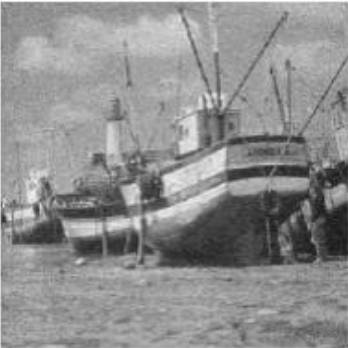

$\mathrm{D}=1.3$

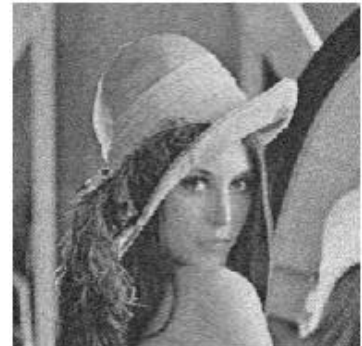

$\mathrm{D}=1$

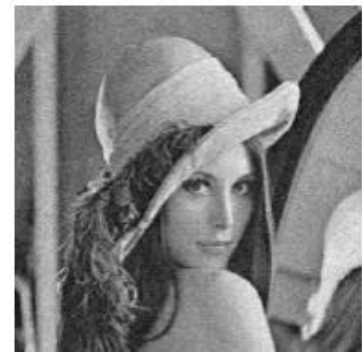

$\mathrm{D}=1.5$

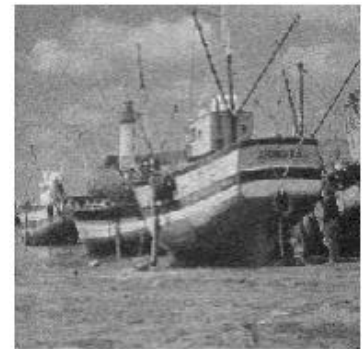

$\mathrm{D}=1$

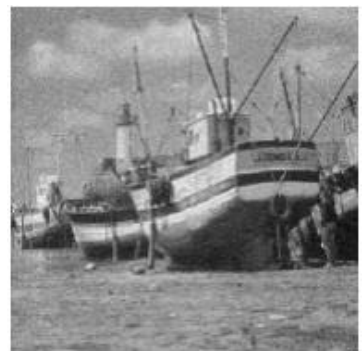

$\mathrm{D}=1.5$

Figure 8. Comparison of the visual quality of the images lena, boat, cameraman and building for different values of $D$ 


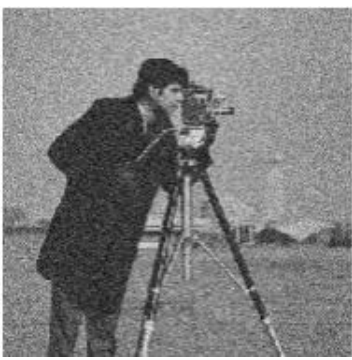

Noisy image

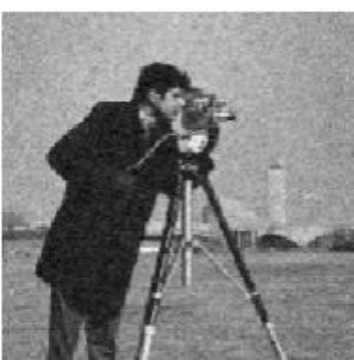

$\mathrm{D}=1.1$

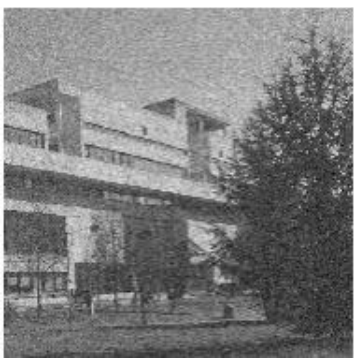

Noisy image

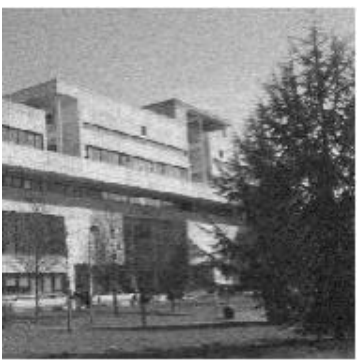

$\mathrm{D}=1.1$

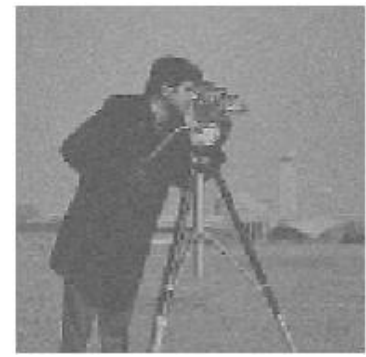

$\mathrm{D}=0.8$

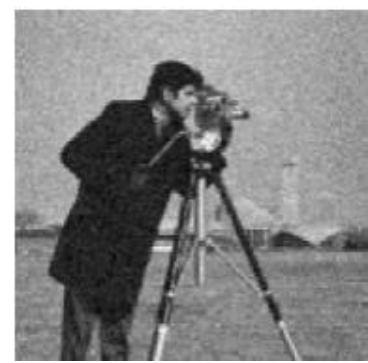

$\mathrm{D}=1.3$

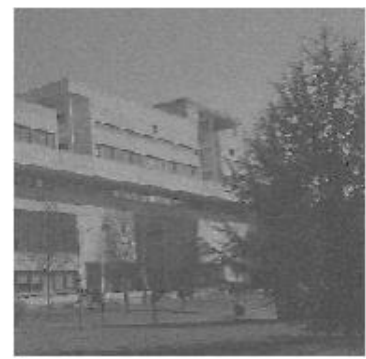

$\mathrm{D}=0.8$

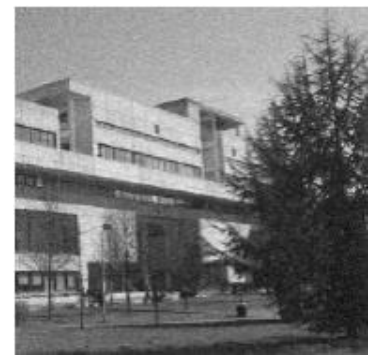

$\mathrm{D}=1.3$

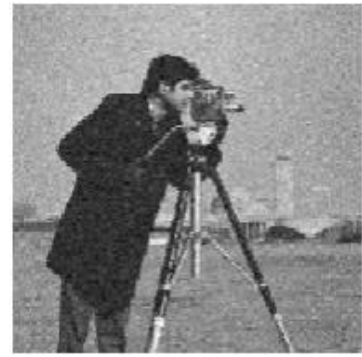

$\mathrm{D}=1$

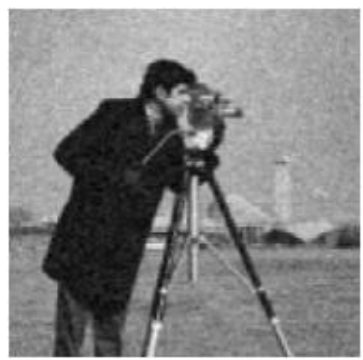

$\mathrm{D}=1.5$

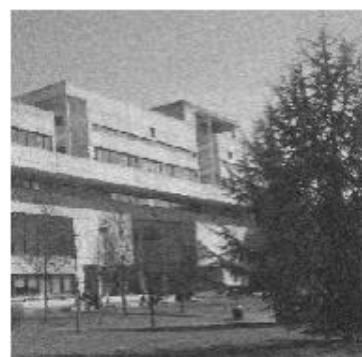

$\mathrm{D}=1$

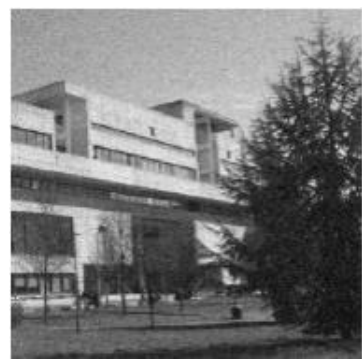

$\mathrm{D}=1.5$

Figure 8. Comparison of the visual quality of the images lena, boat, cameraman and building for different values of $D$ (continue)

\section{CONCLUSION}

In this paper, we have introduced a generalization of the Haar wavelet using fractional operators, where the construction is based on exploiting the FD $Z^{-D}$, approximated by an FIR filter. Variation of the parameter $D$ provides greater flexibility for the fractional filters which constitute the wavelet, leading to more precise decomposition (in terms of detail and approximation). To illustrate the effectiveness of the proposed fractional wavelet (generalized Haar), we have presented the results of using a denoising application on images. The results are very promising, and this idea can be expanded to generalize other families of wavelets. 


\section{REFERENCES}

[1] Michel Misiti, Yves Misiti, Georges Oppenheim and Jean-Michel Poggi, "Wavelets and their applications," ISTE, 2007.

[2] Y. Meyer, "Wavelets and operators," Advanced mathematics. Cambridge University Press, 1992.

[3] S. Mallat, "Multi-resolution approximations and wavelet orthonormal bases of $L^{2}(\mathbb{R})$," Trans. Am. Math. Soc., vol. 315 , no. 1 , pp. 69-87, sep. 1989.

[4] S. G. Mallat, "Multifrequency channel decompositions of images and wavelet models," in IEEE Transactions on Acoustics, Speech, and Signal Processing, vol. 37, no. 12, pp. 2091-2110, Dec. 1989.

[5] S. G. Mallat, "A theory for multiresolution signal decomposition: the wavelet representation," in IEEE Transactions on Pattern Analysis and Machine Intelligence, vol. 11, no. 7, pp. 674-693, July 1989.

[6] I. Daubechies, "Orthonormal bases of compactly supported wavelets," Com. on Pure Appl. Math., vol. 41, pp. 909-996, nov. 1988

[7] D. Mendlovic, Z. Zalevsky, D. Mas, J. Garcia, and C. Ferreira, "Fractional wavelet transform," Appl. Opt., vol. 36, pp. 4801-4806, 1997.

[8] Tao R, Deng B, Wang Y, "Research progress of the fractional Fourier transform in signal processing," Sci China Ser F-Inf Sci, vol. 49, pp. 1-25, 2006.

[9] Huang Y, Suter B, "The fractional wave packet transform," Multidim Sys Signal Process, vol 9, pp 399-402, 1998.

[10] M. Unser, T. Blu, "Fractional splines and wavelets," SIAM Review, vol. 42, pp. 43-67, 2000.

[11] M. Feilner, M. Jacob and M. Unser, "Orthogonal quincunx wavelets with fractional orders," Proceedings 2001 International Conference on Image Processing (Cat. No.01CH37205), Thessaloniki, Greece, vol. 1, pp. 606-609, 2001.

[12] Linfei Chen, Daomu Zhao, "Optical image encryption based on fractional wavelet transform," Optics Communications, vol. 254, pp. 361-367, 2005.

[13] Jun Shi, Nai Tong Zhang, Xiao Ping Liu, "A novel fractional wavelet transform and its applications," Sci China Ser F-Inf Sci, vol. 55, pp. 1270-1279, 2012.

[14] J. Shi , X. Liu, N. Zhang, "Multiresolution analysis and orthogonal wavelets associated with fractional wavelet transform," Signal Image Video Process, vol. 9, pp. 211-220, 2013.

[15] Gaurav Bhatnagar, Q.M. Jonathan Wu, Balasubramanian Raman, "Discrete fractional wavelet transform and its application to multiple encryption," Information Sciences, vol. 223, pp. 297-316, 2013.

[16] Dai Hongzhe, Zheng Zhibao, Wang Wei, "A new fractional wavelet transform," Communications in Nonlinear Science and Numerical Simulation, vol. 44, pp. 19-36, 2016.

[17] T. I. Laakso, V. Valimaki, M. Karjalainen and U. K. Laine, "Splitting the unit delay [FIR/all pass filters design]," in IEEE Signal Processing Magazine, vol. 13, no. 1, pp. 30-60, Jan. 1996.

[18] M. Vetterli, J. Kovacevic. "Wavelets and Subband Coding”. Prentice-Hall, Englewood Cliffs, New Jersey, 1995.

[19] M. Vetterli and C. Herley, "Wavelets and filter banks: theory and design," in IEEE Transactions on Signal Processing, vol. 40, no. 9, pp. 2207-2232, Sept. 1992.

[20] I. Daubechies, "Ten Lectures on wavelets," Springer-Verlag, 1992.

[21] P. Tchamitchian, "Biorthogonalité et théorie des opérateurs," Revista Mathemática Iberoamericana, vol. 3, pp. 163-189, 1987.

[22] S. Mallat. "A wavelet tour of signal processing". Second edition, Academic Press, 1998.

[23] A. Croisier, D. Esteban, and C. Galand, "Perfect channel splitting by use of interpolation/decimation/tree decomposition techniques," In Int. Conf. on Inform. Sciences and Systems, pages 443-446, Patras, Greece, August 1976.

[24] R. Coifman, D. Donoho, "Time-invariant wavelet denoising," in: A. Antoniadis, G. Oppenhiem, Lecture Notes in Statistics, vol. 103, pp. 125-150, Springer, Berlin, 1995.

[25] D. Donoho, I. Johnstone, "Adapting to unknown smoothness via wavelet shrinkage," J. Amer. Statist. Assoc, vol. 90, pp. 1200-1244, 1995. 